\title{
Tecnología y pensamiento futuro del trabajo desde la ergonomía en momentos de crisis global
}

\author{
Technology and future ways of thinking related to work from \\ ergonomics points of views in moments of global crisis
}

\author{
David E. Puentes-Lagos y Gabriel García-Acosta
}

Laboratorio de Ergonomía y Factores Humanos. Escuela de Diseño Industrial, Universidad Nacional de Colombia. Bogotá. depuentesl@unal.edu.co

Recibido 8 Octubre 2009/Enviado para Modificación 20 de mayo 2010 /Aceptado 26 de Enero 2011

\section{RESUMEN}

Este artículo tiene como objeto explorar dos formas de pensamiento respecto a las condiciones futuras de trabajo, en relación con las crisis del trabajo de principios de siglo XXI. Estas dos formas de pensamiento futuro guiaron la investigación y apuntan a una tesis de acción donde las condiciones de trabajo futuro se esperan y una tesis de reacción donde las condiciones de trabajo futuras se construyen. Ahora bien, desde disciplinas como la sociología y la economía se han creado múltiples formas de ver al pasado tecnológico para reconstruirlo, pero, observar hacia el futuro requiere otro tipo de mirada. Disciplinas como la ingeniería y el diseño que trabajan sobre los estudios del futuro y el pensamiento futuro, permitieron empujar esta investigación, con el propósito de generar alternativas para el pensamiento.

Palabras Clave: Tecnología de trabajo, condiciones de trabajo, ergonomía, tecnología, prospectiva, innovación tecnológica (fuente: DeCS, BIREME)

\section{ABSTRACT}

Is it possible to establish (at short, medium and long term) future work conditions or expected work conditions for Colombian people considering upcoming work technologies? Is it possible to anticipate future work desirable work conditions for Colombian people in order to plan (foresee?) work technologies? These questions guided this research and they point to an action thesis and to a reaction one in this context of work crisis. Even though a work technology establishes where, when, how, who, who with, and using what element work is done, it also establishes certain work conditions. Besides, multiple forms of considering and deconstructing past have been created from many disciplines. However, in order to foresee or construct work technologies requires a different perspective for looking further. This 
research has been carried out considering other disciplines points of view regarding Future Studies and Future Thinking Studies. This research has the purpose of finding future paths for Future Thinking Studies from ergonomics point of view in this moment of global work crisis we are going through.

Key Words: Human engineering, technology, forecasting, scientific innovations, ergonomics (source: Mesh, NLM).

$\mathrm{E}$ presente artículo desarrollará algunas de las implicaciones que tiene estructurar el pensamiento futuro de trabajo basado en dos planteamientos. El primero que privilegia la anticipación buscando la reacción ante las tecnologías venideras, y el segundo que propicia el plan y la acción para la proyección de las tecnologías de trabajo futuro con base en principios como la voluntad y la libertad. El ejemplo de la innovación en la comunicación acompañará también estos planteamientos.

En este contexto, luego de la invención del teléfono monedero y antes de la masificación de la telefonía móvil un habitante de metrópoli, requería dos objetos para comunicarse; una agenda y unas monedas. Poner estas en el mercado, requirió de la interacción de vidas e instituciones para poner teléfonos monederos y comunicar dos personas. A mediados del siglo XX, los bolsillos contenían más monedas que hoy y por lo tanto, el fabricante de agendas descubrió que con el cuero sobrante de la agenda, podía hacer monederos para cargar monedas. Hoy a comienzos del siglo XXI, el mismo habitante en Bogotá, no busca su agenda, un teléfono público monedero y unas monedas, porque lleva una innovación tecnológica consigo; un teléfono móvil importado que incluye agenda y otras aplicaciones. Lo carga en un estuche de cuero, elaborado posiblemente de manera local, por el antaño fabricante de agendas.

El teléfono móvil como innovación tecnológica, trae consigo cambios en las dimensiones cultural, económica y política, de la estructura social existente, generando ciclos de expansión y contracción que han sido estudiados principalmente desde la economía; ciclos de crisis que aparentemente se repiten en forma regular (1). En cambio, lo que para la estructura social se presenta como crisis y que estudia principalmente la economía, para los estudiosos de la tecnología se presenta como un ramillete de oportunidades para pensar un futuro diferente. 
Ahora bien, ¿cómo pensar para el futuro una tecnología, que no traiga crisis consigo? Al respecto, en la revisión documental de esta investigación acerca del pensamiento futuro del trabajo, se encontraron dos planteamientos principales.

Un primer planteamiento, corresponde a un proceso histórico y dialéctico del pasado con el presente. Con base en el conocimiento de este proceso se proyecta el futuro. Tiende a marcar una gran admiración por los cambios del presente, e indagar sobre los cambios del pasado para proyectarlos al futuro (2-4). Las respuestas para el futuro en este planteamiento, se encuentran principalmente en el pasado y su encadenamiento con el presente.

El segundo planteamiento busca estimular el proceso de construcción del futuro basado en los principios de libertad, voluntad y acción creadora (5), $(6,7)$. Genera por si mismo fe y esperanza en el futuro mismo desde el presente. Las respuestas para el futuro en este planteamiento, se encuentran en los deseos para el futuro a largo mediano y corto plazo y su articulación para hacerlo posible y viable, concatenando este futuro con el presente.

Crisis del trabajo, oportunidad para anticipar o proyectar tecnologías de trabajo

En la publicación El Trabajo Futuro y El Futuro del Trabajo, Neffa y de la Garza, analizan la preocupación de la sociología moderna por la crisis del trabajo (8). La refieren en América Latina bajo tres aspectos: el aumento de la economía no estructurada, el autoempleo, y la precarización de las condiciones de trabajo. Si bien los dos primeros aspectos que se plantean, son quizás los más relevantes para salir de la crisis, estos son aspectos macroeconómicos difíciles de intervenir desde una sola perspectiva disciplinar. Ahora bien, el tercer aspecto de las condiciones de trabajo se presenta más viable y factible en las áreas disciplinares preocupadas por el problema de la Salud y la Seguridad en el Trabajo como la Ergonomía, disciplina desde la que se plantea este artículo.

Así por ejemplo, las condiciones de trabajo desde la Ergonomía, son definidas por Wisner, como un campo extenso y comprende la duración del trabajo en jornadas, semanas, años, en fin, en la vida; incluye contratación, desvinculación y a menudo, el salario y los beneficios sociales. Incluye también aspectos de Salud, Higiene Industrial, la Seguridad Industrial, la sobrecarga y la sub-carga Psicosocial (9). 
Pero, a diferencia de lo que nos revela Neffa como crisis en el trabajo desde la sociología, para la tecnología, la crisis se presenta como una oportunidad de pensar el trabajo de otra manera. La innovación en el trabajo, repensar el trabajo con base en el pensamiento futuro. Ahora bien, el pensamiento futuro y su potencial uso para el pensamiento del trabajo del futuro, puede hacerse gracias a los instrumentos desarrollados desde las áreas que trabajan en el desarrollo tecnológico. Son diversos sus medios y fines, así como los campos de aplicación. Estos instrumentos entran dentro del espectro de conocimientos denominados, estudios sobre el futuro (Tabla1).

Tabla 1. Algunas técnicas y métodos usados dentro de los estudios sobre el futuro. Compilación y adaptación del autor

\begin{tabular}{|c|c|c|c|}
\hline $\begin{array}{l}\text { Modelos, métodos y } \\
\text { técnicas }\end{array}$ & Estructura y uso & $\begin{array}{l}\text { Ejemplo y campo de } \\
\text { aplicación }\end{array}$ & Partes interesadas \\
\hline Lluvia de ideas & $\begin{array}{l}\text { Presentar múltiples posibilidades en la } \\
\text { solución de un problema, sin importar } \\
\text { su viabilidad o probabilidad. La ideas } \\
\text { más potenciales son a veces las que } \\
\text { en primera instancia parecen menos } \\
\text { viables }\end{array}$ & $\begin{array}{l}\text { Búsqueda de nuevas ideas } \\
\text { originales. Para la búsqueda } \\
\text { de conceptos de diseño para } \\
\text { nuevos productos y servicios. }\end{array}$ & $\begin{array}{l}\text { Múltiples, según el } \\
\text { problema tratar. }\end{array}$ \\
\hline $\begin{array}{l}\text { Modelos de pronóstico } \\
\text { cuantitativos }\end{array}$ & $\begin{array}{l}\text { Tomar datos históricos y periódicos } \\
\text { de variables, que son sometidos a } \\
\text { modelos de series de tiempo como } \\
\text { regresiones, mínimos cuadrados y } \\
\text { modelos robustos, que permiten hacer } \\
\text { una proyección con un nivel de con- } \\
\text { fianza porcentual }\end{array}$ & $\begin{array}{l}\text { Riesgos de inversión en el } \\
\text { sector financiero, proyección } \\
\text { de las tasas de interés y creci- } \\
\text { miento económico. }\end{array}$ & $\begin{array}{l}\text { Actividades } \\
\text { económicas sociedad } \\
\text { en general. }\end{array}$ \\
\hline Estudios Delphi & $\begin{array}{l}\text { Iteración de intereses políticos contra } \\
\text { potencial de desarrollo en políticas } \\
\text { de países para la inversión. Encuesta } \\
\text { realizada a expertos en diferentes } \\
\text { áreas de desarrollo y diferentes nive- } \\
\text { les de decisión en una sociedad }\end{array}$ & $\begin{array}{l}\text { Estrategia para el desarrollo } \\
\text { tecnológico en China 2020- } \\
2025 \text {. Estrategias de ciencia } \\
\text { y tecnología, I + D, tanto en } \\
\text { empresas como en países. }\end{array}$ & $\begin{array}{l}\text { El estado, las } \\
\text { empresas en el } \\
\text { mercado de bienes y } \\
\text { servicios, la sociedad }\end{array}$ \\
\hline Vigilancia tecnológica & $\begin{array}{l}\text { Revisión sistemática y bajo modelos } \\
\text { matemáticos de variables signifi- } \\
\text { cativas que afecten el desarrollo y } \\
\text { conocimiento aplicado en un material, } \\
\text { un proceso productivo en relación con } \\
\text { innovación en el mercado de bienes } \\
\text { y servicios }\end{array}$ & $\begin{array}{l}\text { Estudio de los objetos en el } \\
\text { Hogar, España. Fundación } \\
\text { OPTI para la prospectiva } \\
\text { tecnológica. }\end{array}$ & $\begin{array}{l}\text { Empresarios y } \\
\text { academia que } \\
\text { participa en el } \\
\text { desarrollo de nuevos } \\
\text { materiales y proceso } \\
\text { como apoyo a la } \\
\text { ciencia normal }\end{array}$ \\
\hline $\begin{array}{l}\text { Prospectiva } \\
\text { tecnológica }\end{array}$ & $\begin{array}{l}\text { Establecer futuros posibles, probables } \\
\text { y deseables en un producto o proceso } \\
\text { en una empresa. Bajo la condición } \\
\text { que sean múltiples }\end{array}$ & $\begin{array}{l}\text { El desarrollo del VHS en lugar } \\
\text { del BETA. Busca ordenar las } \\
\text { prioridades en el momento de } \\
\text { desarrollar una tecnología. }\end{array}$ & $\begin{array}{l}\text { Empresas y cadena } \\
\text { tecnológicas. }\end{array}$ \\
\hline $\begin{array}{l}\text { Planificación por } \\
\text { escenarios }\end{array}$ & $\begin{array}{l}\text { Planteamiento mediante relatos cortos } \\
\text { y una idea fuerza acerca del futuro de } \\
\text { una empresa, un país o un conflicto. } \\
\text { La validez está condicionada a un } \\
\text { escenario factible y otros alternativos }\end{array}$ & $\begin{array}{l}\text { Escenarios de Montfleur en } \\
\text { Sudáfrica. Destino Colombia, } \\
\text { en busca de una salida a la } \\
\text { crisis (1997) }\end{array}$ & $\begin{array}{l}\text { Múltiples, según el } \\
\text { problema tratar }\end{array}$ \\
\hline
\end{tabular}

El pensamiento futuro, se nos presenta de esta forma, como una solución que permite modelar el futuro y el trabajo futuro. La administración, las artes, la arquitectura, la ingeniería y el diseño, son quizás las disciplinas 
que más utilizan el pensamiento futuro, para ir más allá en el tiempo, explorando el ramillete de posibilidades de innovación, tratando de encontrar las pistas sobre la solución a las problemáticas del presente hacia el futuro y viceversa. El pensamiento futuro es el comienzo y el fin, el medio y el resultado, la causa y la consecuencia misma de la producción de estas disciplinas.

Respecto a lo antes mencionado, algunos productos del pensamiento futuro, son estrategias, dibujos, maquetas y modelos: conducen de una forma al diseño del futuro más no del futuro mismo porque es indeterminado (10). Para las disciplinas que desarrollan y trabajan con base en el pensamiento futuro, el futuro se diseña y se construye por medio de un proyecto. El diseño es un vocablo derivado del italiano "disegno", dibujo, designio, lo por venir, la visión y la estrategia del futuro representada de múltiples formas (11). El producto de la actividad del diseño como obra, es un plan de lo que está por hacerse, es el proyecto de lo que estamos pensando en hacer con el futuro.

El acto de diseñar como prefiguración, es el proceso previo en la búsqueda de una o múltiples soluciones a un conjunto de condiciones que se han presentado, para soñar con hacer lo que aún no está. A esta forma de pensamiento algunos autores la denominan Meta-cognición, otros la definen como pensamiento de diseño (12). Es desde este pensamiento de diseño y pensamiento futuro, que buscamos una salida al problema de la crisis del trabajo y el cómo lo hemos conocido hasta ahora. En este sentido, la contribución del pensamiento futuro, considerada en esta investigación, apuntó a modos de llegar a los futuros esperados y deseados conocidos como futuribles.

De acuerdo con la prospectiva, disciplina donde se agrupan algunos de los estudios sobre el futuro, un futurible para es uno de los tantos futuros posibles y deseables que se presenta cuando se piensa en futuro (6). Estos modos de aproximar el pensamiento futuro se hicieron desde la ergonomía por su cercanía al diseño, y por ser una de las áreas que trabaja en el mejoramiento de las condiciones futuras de los trabajadores.

La tecnología de trabajo, una manera de acercarse al pensamiento futuro del trabajo desde la ergonomía. 
En la ergonomía, reconocida por su famoso postulado de adaptar el trabajo al hombre y no el hombre al trabajo, existe una gran cercanía al diseño y por tanto al pensamiento futuro. Sus escuelas anglosajona, nórdicafrancesa y oriental dan prueba de ello, por su aplicación en el diseño de productos, servicios y procesos, donde el pensamiento futuro, es una parte de su actividad intelectual. Es evidente en sus planteamientos recientes, el énfasis en el carácter prospectivo; aportes hechos desde la administración, la ingeniería y el diseño ${ }^{1}$.

Hoy la ergonomía cuenta con áreas teóricas que incluyen pensamiento futuro de forma implícita como: la Ergonomía de Concepción, la Macroergonomía y la Antropotecnología.

Surge entonces la necesidad de acercar de manera explícita la ergonomía al pensamiento futuro del trabajo, de cara a momentos de crisis y los cambios de las tecnologías en periodos de tiempo cada vez más cortos. Para ello se hizo necesario desarrollar, una noción para viajar en el espacio y el tiempo integrando de manera explícita el pensamiento futuro, que conectara a la tecnología y al trabajo. Tecnología de trabajo o tecnología en situación de trabajo, es una noción que permite ver al trabajo y la tecnología unidos, para que sean anticipados o proyectados hacia el futuro; con esta noción se buscó facilitar la anticipación o la proyección de las condiciones de los trabajadores del futuro desde la ergonomía. Es de resaltar que la noción tecnología de trabajo, tiene como principal propósito, facilitar el pensamiento futuro del trabajo, pero fue construida a partir de múltiples nociones de tecnología y de trabajo consultadas, que han tenido en cuenta el espacio, el tiempo y el individuo de manera agregada, sin separar sus componentes.

Con base en esta noción, fueron dos preguntas, las que guiaron el proceso de investigación para el pensamiento futuro del trabajo y de las condiciones de los trabajadores: ¿Podemos proyectar las condiciones de trabajo futuras o esperadas de los colombianos, según las tecnologías de trabajo venideras en el corto, mediano y largo plazo?, ó, ¿podemos construir las condiciones futuras de trabajo que deseamos para los colombianos, proyectando las

${ }^{1}$ Puentes D. [Tecnología y Prospectiva en el Trabajo: Aproximación a un pensar futuro del trabajo desde la Ergonomía]. Tesis de Maestría en Salud y Seguridad en el Trabajo. [2009]. Se localiza en: Facultad de Enfermería. Bogotá: Universidad Nacional de Colombia, Sede Bogotá. 
tecnologías de trabajo? Las respuestas apuntalan como resultado, una tesis de reacción y otra tesis de acción: ambas en las tecnologías de trabajo.

Grafico 1. Dos tesis para guiar el pensamiento futuro del trabajo desde la ergonomía
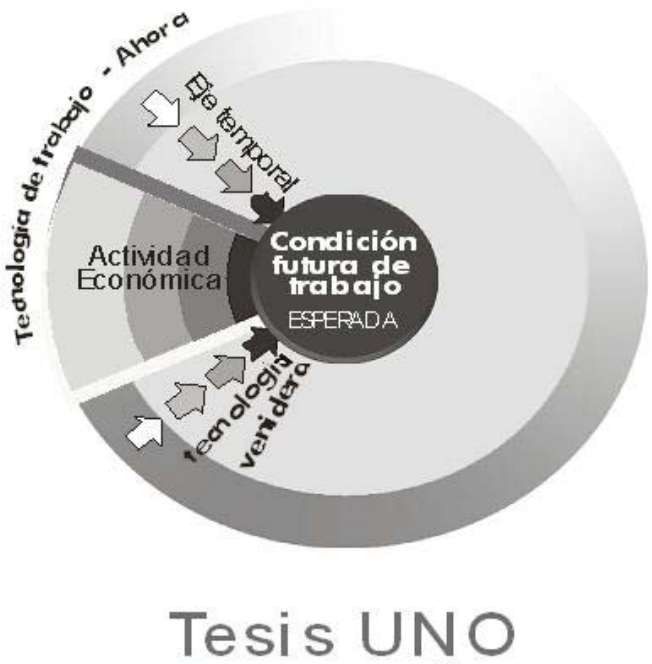
REACCIÓN
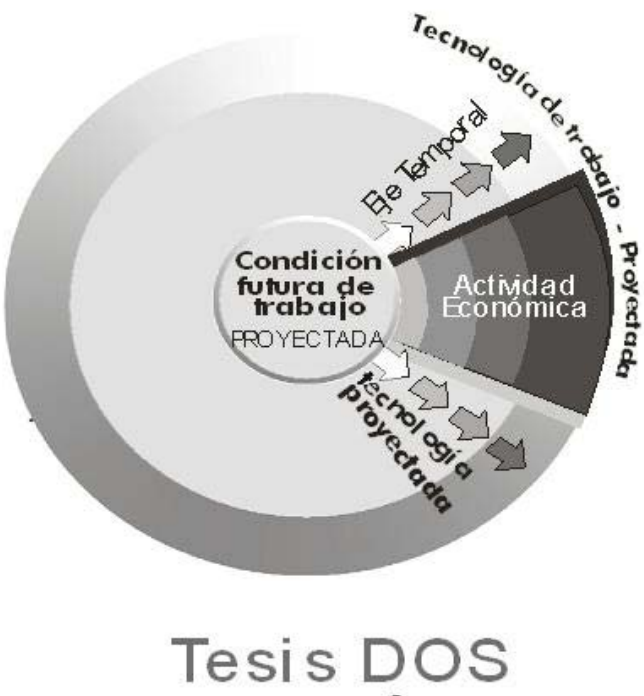
ACClÓN

Fuente: Tesis de Maestría-Tecnología y Prospectiva en el trabajo

Se evidencian entonces dos tesis con base en las dos preguntas: una donde las condiciones de trabajo futuras son esperadas porque la tecnología apenas se anticipa, y otra donde las condiciones de trabajo se construyen porque la tecnología se proyecta.

No obstante lo anterior, para comprender las condiciones de los trabajadores colombianos en las tecnologías de trabajo del futuro, es necesario establecer la situación actual de los colombianos. Se usó para ello, el Índice de Adelanto Tecnológico que se encuentra dentro de la medición del Índice de Desarrollo Humano que compara los países. Si bien el índice hace énfasis en "la era de las redes" y mide logros y no intenciones, esfuerzos y contribuciones colaterales, es un indicador reflejo de capacidades y posibilidades de Colombia y los colombianos. 
Colombia, para el año 2003 ocupó el puesto número 47 y es clasificado como seguidor dinámico. Este índice refleja entre otros: 5,3 años de escolaridad formal promediada, 40 patentes registradas por colombianos en el 2000, matriculación de 5,2 \% en educación terciaria dentro de las "big science", ingenierías y diseños.

Pero, insistimos en mirar este índice como potencial y no como problema. Cada país cuenta con sus propias tecnologías de trabajo y sus problemas, que corresponden con su propio desarrollo tecnológico. En los países de la "América Ladina", transcultural, ambigua y fronteriza que nos presenta Guillermo Restrepo (14), es posible que las características de nuestras tecnologías de trabajo o tecnologías en situación de trabajo, deban enfrentar la crisis integrando la pasión creadora al servicio del saber, la sabiduría de la vida y para la vida, y no quedarse en un simple conocimiento científico aplicado, al que solo pueden acceder los países con mayor educación formal y terciaria en las "big science", ingenierías y diseños.

De otro lado, una tecnología de trabajo es una tecnología en situación de trabajo bajo múltiples dimensiones y componentes, que generan dinamismo entre sus componentes como lo muestra el gráfico 2 .

Grafico 2. Dimensiones y componentes dimensionales de las tecnología de trabajo

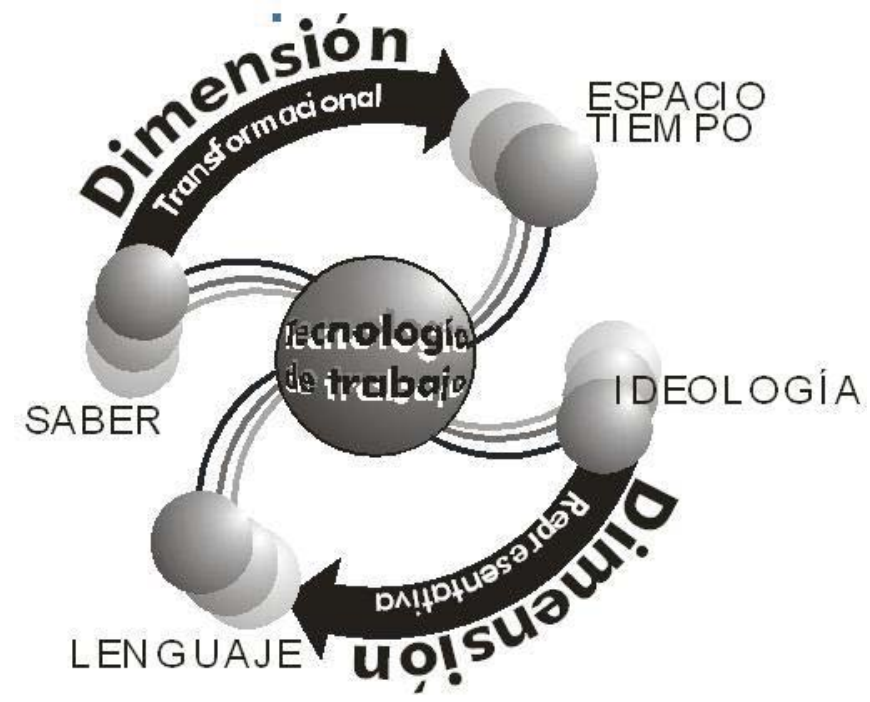

Fuente: Tesis de Maestría- Tecnología y Prospectiva en el trabajo 
En este punto, podrían establecerse ciertas similitudes con los estudios sociológicos de la tecnología, donde las grandes tendencias son el determinismo tecnológico y la construcción social de la tecnología (15), (16). No obstante la mayoría de sus planteamientos son socio-históricos. Por otro lado en cambio, en la visión prospectiva de la noción tecnología de trabajo, la transformación y la representación, son las dimensiones que además de generar una dinámica o mutación tecnológica (17), permiten diferenciar las tecnologías de trabajo entre sí, según el grado de transformación del presente hacia el futuro y viceversa.

La dimensión transformacional, es relativa al saber y al espacio-tiempo; define la capacidad de transformar las sustancias, como una metafísica de la tecnología. Tecnología para saber transformar el espacio-tiempo. En el ejemplo de la introducción, permite al fabricante de cuero transitar de la agenda al monedero.

La dimensión representativa, es relativa a la ideología y el lenguaje, que define en cambio, la capacidad de representar los ideales de vida y comunicación de un colectivo; lo cognoscitivo del vivir y convivir. Tecnología de un lenguaje que representa los ideales de un individuo o un colectivo. En el ejemplo de la introducción, se observa en el paso de la telefonía análoga y su lenguaje de impulsos eléctricos, a la telefonía digital de transporte inalámbrico de datos.

Ambas dimensiones conviven, y pensar la tecnología de trabajo hacia el futuro, demanda verlas y comprenderlas de manera simultánea y "multidimensionalmente" (en múltiples dimensiones y escalas). En el ejemplo se debe ver simultáneamente, la transformación de los artefactos para la comunicación, como el estilo de vida y la ideología detrás de todo el sistema de comunicación.

Recapitulando, luego de una caracterización tecnológica colombiana y la aproximación a la noción de tecnología de trabajo o tecnología en situación de trabajo, nos queda entrar en la tarea de anticipar o proyectar hacia el futuro. Pero antes, se debe comprender que parte de la crisis de los países latinos, se presenta quizás porque el pensar la tecnología del futuro, ha sido renunciar casi absolutamente a construir las propias tecnologías de trabajo, resolviendo el problema con aparatos y artefactos, que aparecen de la nada, convirtiendo a Colombia y los países de la zona 
en consumidores de "cacharros" encargados a otros. Esta renuncia a la construcción de tecnologías de trabajo, convierte estos países en simples consumidores, que han sido parte de un pensamiento futuro desde países adelantados tecnológicamente, no como creadores, sino como mercado objetivo de tecnologías de trabajo, y, en el mejor de los casos, como receptores de transferencias de tecnologías de trabajo, estudiadas por la antropotecnología (9).

Ante este panorama, es prioritario pensar el futuro con alternativas que generen una mayor actividad creadora para el futuro, por parte de Colombia y los países latinos.

Acción y Reacción dos tesis para pensar el futuro del trabajo.

A partir de la relación entre la crisis y las tecnologías de trabajo, la investigación desarrolló el planteamiento de dos alternativas opuestas para pensar las condiciones de trabajo del futuro desde la ergonomía, una de reacción y otra de acción.

Tesis uno: De Reacción-Anticipar las condiciones de trabajo desde la ergonomía, con base en las tecnologías de trabajo esperadas. Después de la masificación de la invención del teléfono móvil y antes de una nueva invención, toda la industria de la comunicación de un país como Colombia, que construye pocas tecnologías de trabajo con lenguaje digital, genera una reacción, para participar en el nuevo modelo de producción y consumo que trae consigo, esta innovación en la comunicación. En el nuevo paradigma (18), reaccionan todas las variables que en los teléfonos de monederos tenían un gran protagonismo. Reaccionan las personas, disminuyendo la cantidad de circulante en monedas, desapareciendo las agendas tradicionales, y fabricando bolsillos para los pantalones que sean más livianos, porque soportarán menos carga. En conclusión, reacciona toda la tecnología de trabajo, alrededor de la comunicación y, las variables que antes fueron vitales, pasaron a tener un rol diferente y más trivial, trayendo consigo y por consecuencia, un cambio en las condiciones de trabajo de todas las industrias de la comunicación.

La tesis de reacción apunta en el mejor de los casos a la anticipación de las tecnologías de trabajo y, en el peor, a la simple adaptación de las tecnologías de trabajo. En el caso de la anticipación, se podrían anticipar 
las variables vulnerables de las tecnologías de trabajo que se encuentran por venir, y gracias a ese análisis preparar posibles escenarios de llegada y modelar con ellos las condiciones de trabajo esperadas. En ese caso, revisando el cuadro sobre los estudios de futuro (tabla1), métodos, como la lluvia de ideas y la planificación por escenarios serían significativamente útiles. Pero, en el caso de la adaptación de las tecnologías de trabajo, los modelos de pensamiento futuro, son poco útiles, se basarán más en la evidencia de problemas de compatibilidad e interacción en las tecnologías de trabajo. Estas evidencias estarán ya materializados en personas accidentadas, enfermas, problemas de productividad, calidad, incluso severos daños ambientales o en las instalaciones de trabajo.

Desde la ergonomía, cuando se piense en anticipación, se pensaría en soluciones para la adecuación física, ambiental, cognitiva y organizacional, de las tecnologías de trabajo por venir, previendo con base en las variables de quién (dimensión representativa), y cómo (dimensión transformacional), las pensaron. Esto ayudará en el proceso de adecuación de lo que está por venir para un trabajador colombiano. En este sentido, el planteamiento más cercano es la antropotecnología, que con base en información clara sobre las dimensiones de las tecnologías de trabajo, puede pronosticar algunos problemas de compatibilidad en una transferencia de tecnología. En el caso de la adaptación de las tecnologías de trabajo, los planteamientos de la ergonomía de corrección, son quizás los más cercanos e irónicamente, los que más comúnmente se presentan en el campo empírico, como la industria de la comunicación con base en telefonía móvil que genera difíciles condiciones de trabajo.

Este tipo de pensamiento se corresponde más con modelos basados en mirar los hechos, en diagnosticar y en prevenir, más que en explorar en el futuro del tiempo, pero su importancia por estar en el día a día del trabajo de un ergónomo y mostrar con hechos sus resultados positivos, obliga a futuras investigaciones, para reforzar la tradicional importancia de un buen diagnóstico de las condiciones de trabajo.

Las condiciones de trabajo en estos casos, son esperadas y no construidas. En esta tesis se podría, con base en las cargas de trabajo esperadas, estudiar jornadas de trabajo, los pagos, las condiciones de seguridad, de higiene, de salud, psicosociales y demás elementos que determinarán la condición futura de trabajo, permitiendo mejorar la vida y la calidad de 
vida de los trabajadores, pero también, la productividad y las demandadas en el consumo y producción de bienes y servicios. Este es un escenario donde el control y el poder de quienes hacen el desarrollo tecnológico en el mundo, determina la dimensión transformacional y representativa de la tecnología. En esta tesis, cuando la ergonomía de corrección y la antropotecnología son utilizadas, se podría modificar en alguna medida la dimensión transformacional de una tecnología de trabajo.

En cambio, resulta más difícil cambiar la dimensión representativa, porque el lenguaje y la ideología de esas tecnologías de trabajo, se corresponden con el país creador y son diferentes al país que las consume. Quizás este es el caso local y una buena parte de "América Ladina" (14), que solo consume tecnologías de trabajo creadas en otros países. Aspectos como la baja educación formal y terciaria, capacidad inventiva limitada mantienen y mantendrán un tiempo ese consumo.

En esta tesis sólo se reacciona, por lo tanto, las condiciones de trabajo del futuro serán esperadas y estarán subordinadas a la ideología y lenguaje de quienes pensaron desde la dimensión representativa las tecnologías de trabajo del futuro, con el peligro de adquirirla en forma de "cacharro" en el mercado.

Si bien esta tesis tiene problemas complejos, para ver la situación de manera positiva, se debe aclarar que hay múltiples formas de reaccionar. Algunos de los mejores tenistas profesionales del mundo, desarrollan el arte de la reacción y la defensa, para convertir el ataque de un contrario, en acción ofensiva propia. El pensamiento futuro del trabajo desde la ergonomía, tendría que desarrollar el arte de convertir tesis de reacción, en tesis de acción: continuar con futuras investigaciones como la de este artículo, es prioritario.

Tesis dos: De Acción - Construir las condiciones de trabajo desde la ergonomía, con base en las tecnologías de trabajo que se proyectan. Las condiciones de trabajo futuras, de las tecnologías de trabajo proyectadas con base en la telefonía móvil, se piensan desde los países que lideran la investigación y desarrollo alrededor de las tecnologías de la información y la comunicación. Estos países líderes en innovación tecnológica, como Finlandia, Norteamérica y Japón, definirán el futuro de la telefonía móvil. El país y las empresas líderes actúan estableciendo como serán 
los requerimientos y determinantes de la tecnología de trabajo del futuro proyectadas. La proyección es un plan que posteriormente se ejecuta gracias al saber desarrollado alrededor del problema y la solución, generando un rompimiento paradigmático y una innovación.

En esta tesis priman dos elementos: el plan y la acción. El plan en el caso de las tecnologías de trabajo es el conjunto de estrategias para llegar a un saber para si misma y su fin, además de preparar su estructura de tal manera que pueda actuar. Elaborar el plan y accionarlo, son fortalezas de la tecnología contemporánea. A esta "megamáquina" que descubrió Lewis Mumford alrededor de la tecnología moderna y en este caso de las tecnologías de trabajo (19), le es propio el plan y la acción para construir las condiciones de trabajo futuras. Se evidencia de esta forma que la construcción de la tecnología de trabajo futura, está ligada con la construcción de las condiciones de trabajo futuras y por lo tanto, este debería ser uno de los campos de investigación del pensamiento futuro del trabajo desde la ergonomía.

Por otro lado en la práctica en Colombia, como lo evidencian todas las actividades económicas, registradas en el decreto 1607 de 2002, la construcción de las condiciones de trabajo con base en la proyección de la tecnología hacia el futuro, brilla por su ausencia. Se evidencia también, en la poca producción de bienes de capital y la baja protección industrial de estas ante la Superintendencia de Industria y Comercio para proteger sus invenciones. Dos causas son relevantes: la primera es, actividades económicas con predominio en bienes de consumo, mientras hay poca presencia de producción de bienes de capital. La segunda es el carácter exportador de materias primas con pocas o nulas transformaciones.

En comparación con nuestro adelanto tecnológico, los países líderes tecnológicos duplican la educación formal y quintuplican la terciaria, países como Japón patentan 1000 veces más. Por lo tanto para la construcción de las condiciones de trabajo desde la ergonomía en países líderes tecnológicos, la tarea es más sencilla que para la mayoría de los latinos.

La construcción de las condiciones de trabajo futuras, requiere tener el control sobre lo que se proyecta en las tecnologías de trabajo, para establecer requerimientos y especificaciones de diseño alrededor del cómo se hará un trabajo en el futuro. Obviamente se debe saber en qué vamos a 
trabajar en el futuro, lo que implica decisiones políticas importantes que definan hacia dónde dirigir los esfuerzos tecnológicos de un país entero. Por ejemplo, un estudio Delphi realizado en China en el 2006, para pensar las tecnologías de trabajo con proyección del 2020 al 2025 determinó los siguientes tópicos tecnológicos: 1. Informática electrónica y comunicación, 2. Energía, 3. Nuevos materiales, 4. Manufactura avanzada, 5. Explotación de recursos ambientales, 6. Nuevas sustancias químicas, 7. Exploración del espacio (20). Mientras que el Consejo Privado de Competitividad Nacional en Colombia, estableció como industrias de clase mundial para el 2015 las siguientes: 1. Salud y Ortodoncia, 2. Mantenimiento y Reparación de Aviones, 3. Biocombustibles, 4. Industria Maderera, 5. Biotecnología, 6. Industria Farmacéutica 7. Pequeñas Series (21). Cabe la pena resaltar que mientras un país líder tecnológico, como China, convoca la sociedad para definir colectivamente la tecnología del futuro, Colombia en cambio con un mandato político, delega a un comité privado los tópicos para el desarrollo empresarial.

En este orden de ideas, las tecnologías de trabajo se diferencian entre países y por lo tanto el reto en la tesis de acción sobre el pensamiento futuro desde la ergonomía, es desarrollar conocimientos locales. Mientras los chinos piensan en hacer los aviones y naves espaciales, los colombianos apenas piensan hacer mantenimiento de aviones. Y es allí, donde se presentarán los principales problemas en las condiciones de trabajo futuras, porque el trabajo del futuro en mantenimiento de aviones por ejemplo, está siendo diseñado por países orientales, anglosajones y europeos.

Algunas conclusiones de la tesis de Acción y Reacción y el caso colombiano

Recapitulando las dos tesis desarrolladas podemos establecer un conjunto de conclusiones entre las que se encuentran las siguientes: El que espera y vigila se debe a la reacción y desarrollará estas destrezas condicionando el pensamiento futuro desde la ergonomía a una estrategia reactiva, en la cual prima la defensa: en el mejor de los casos tiende a anticipar y en el peor corregir las condiciones de trabajo. El que ataca en cambio, hace un plan y actúa: tiene la ventaja en el pensamiento futuro desde la ergonomía y por consecuencia en la construcción de las condiciones futuras de trabajo, porque si bien el futuro es indeterminado, quizás por lo menos logre mejorar su conocimiento actual sobre sus posibles caminos. 
En ese sentido, la tesis de construcción de las condiciones de trabajo y pensamiento futuro desde la ergonomía es un poco utópica para Colombia. No obstante creemos que apoyarse en los comités de competitividad, nacionales, regionales y locales, así como algunos estudios de tendencia locales, pueden revelar caminos acerca del futuro y colaborar con la actividad de pensamiento futuro desde la ergonomía. Las prioridades políticas nacionales, deberían apuntar a la construcción de tecnologías seguras, en las que prime la acción, sin dejar de lado una política contra la enfermedad y la accidentalidad, en las que prima mucho más una tesis de reacción.

En este sentido, la ergonomía colombiana tiene mucho camino por recorrer, por las crecientes estadísticas de enfermedad profesional en el sistema de riesgos profesionales, además de la baja capacidad de innovación materializada en pocas patentes y protecciones de diseño. Enfrentará el reto de aprender del pensamiento futuro de otros países líderes tecnológicos y de la dimensión representativa de los que diseñarán las tecnologías de trabajo del futuro.

En relación con la crisis del trabajo que para América Latina y sobre todo la crisis del trabajo en Colombia, que se manifiesta en el aumento de la economía no estructurada, el autoempleo, y la precarización de las condiciones de trabajo, el pensamiento futuro del trabajo desde la ergonomía en las dos tesis planteadas, debe tener un mayor impacto en las condiciones de trabajo. Además, estas también deben contribuir en generar mayor producción de bienes de capital, para estructurar una economía más sólida que traiga consigo una mayor formalización del empleo, condición actual de los países con altos Índices de Adelanto Tecnológico, mayor ingreso per cápita y mejor calidad de vida de sus habitantes

\section{REFERENCIAS}

1. Shumpeter JA. Ciclos económicos: análisis teórico, histórico y estadístico del proceso capitalista: Prensas Universitarias de Zaragoza; 1939.

2. Standage T. El futuro de la tecnología - artículos seleccionados Economist. Buenos Aires: Ed. Cuatro Media; 2008. p. 368.

3. Derry TK, Trevor W. Historia de la tecnología. Vol. 1.6 ed: Siglo XXI; 1960. p. 393.

4. Postman N. Tecnópolis: la rendición de la cultura a la tecnología. Barcelona: Círculo de Lectores; 1994. p. 277.

5. Berger G. Etapes de la prospective. 2 ed. París: Presses Universitaires de France; 1967 p. 343. 
6. Gabiña J. El futuro revisitado: La reflexión prospectiva como arma de estrategia y decisión, México, D.F.: Alfaomega Grupo Editor S.A; 1995.

7. Godet M. De la anticipación a la acción: Manual de prospectiva y estrategia. Paris: Alfaomega- marcombo; 1993.

8. De la Garza TE. Problemas clásicos y actuales de la crisis del trabajo. En: De la Garza TE (Coord.) El futuro del trabajo, el trabajo futuro. Buenos Aires: Ed. Clacso; 2001. pp. 11-31.

9. Wisner A. Ergonomía y Condiciones de Trabajo. Buenos Aires: Ed. Humanitas; 1998. p. 318.

10. Heidegger M. El concepto de tiempo. Madrid: Ed. Trotta; 1995. p. 69.

11. Manzini E. Artefactos. Madrid: Experimenta ediciones de diseño; 1990.

12. Organización de Estados Iberoamericanos para la Educación, la Ciencia y la Cultura [Internet]. Disponible en: http://www.oei.es/. Consultado: Enero 2007.

13. Restrepo G. La promesa de Telémaco. Universitas Humanística. 2008; 65 (1): 49-71.

14. Wyatt S. Technological determinism is dead; long live technological determinism. In: Hackett EJ, Amsterdamska O, Lynch M, Wajcman J( Eds.). The Handbook of Science and Technology Studies, Third edition. Cambridge, London: The MIT Press; 2008. p. 165-180.

15. Klein HK, Kleinman DL. The social construction of technology: Structural considerations. Science, Technology and Human Values. 2002; 27(1), 28-52.

16. Aït-El-Hadj S. Gestión de la tecnología: la empresa ante la mutación tecnológica. Paris: Adison-Wesley Iberoamericana; 1990.

17. Kuhn T. La estructura de las Revoluciones Científicas: Nueva traducción e introducción de Carlos Solís. 3ed. México D.F: Fondo de Cultura Económica; 2006. p. 361.

18. Mumford L. Técnica y civilización. Madrid: Alianza Editoria; 1962. p. 522.

19. Ronping M, Zhonbao R, Sida Y, Yan Q. Technology foresight towards 2020 in China: the practice and its impacts. Technology Analysis \& Strategic Management 20. 2008; (3): 287-307.

20. Comité Privado de Competitividad Colombia [Internet]. Disponible en: http://www.compite. ws/spccompite/content/page.aspx?ID=53. Consultado: diciembre 2008. 